\section{PERFECT FOR TECHNOLOGY AFICIONADOS}

At this year's BDIA Dental Showcase the Oral-B team will be on hand to showcase their latest power toothbrush as well as their Pro-Expert toothpaste.

Technology aficionados will love the new Oral-B SmartSeries electric toothbrush with Bluetooth 4.0 connectivity. The new toothbrush connects to the Oral-B App and records brushing activity, which patients can then share with their dental professional.

Visitors can also find out about the revolutionary Test Drive trial programme, which allows multiple users to experience Oral-B power and toothpaste using a shared handle in a hygienic way. Both professional and patient can now evaluate the product before recommending/buying it.

Excitement will also be gained from the news that for the third consecutive time, the independent, not-for-profit Cochrane Collaboration has concluded that oscillatingrotating technology - used in Oral-B power toothbrushes - is the only type of power brush consistently proven to reduce more plaque and gingivitis versus manual brushing in the short and long term. This outcome was derived from a larger study that concluded power brushes outperform manual toothbrushing. 1

Visit Gold Sponsor Oral-B on J04.

1. Yaacob $M$, Worthington $H V$, Deacon $S A$ et al. Powered versus manual toothbrushing for oral health. Cochrane Database Syst Rev 2014; 6: CD002281.

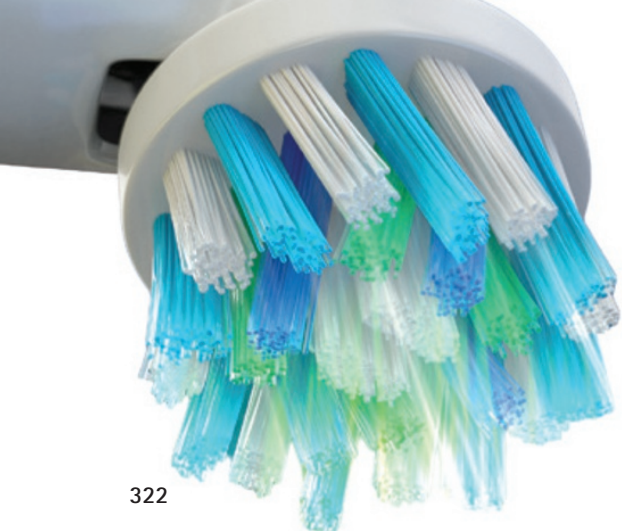

\title{
UNIQUE OPEN INTERFACES BETWEEN DEVICES AND SOFTWARE
}

Visit Plandent on stands G10/H13 for demonstrations of their innovative order systems: PlanORDER, the stock management system for the larger practice which eliminates waste and errors in stock ordering; and PlanORDERlite, the unique ordering app, allowing you to order by the touch of a button from a mobile device.

See Planmeca debut the full range of open CAD/ CAM solutions for dentists and dental labs on stands G08/H12. This consists of Planmeca PlanScan - the first dental unit integrated intraoral scanner for digital 3D impressions; PlanCAD, the open CAD software suite designed especially for dentists which is the perfect tool for sophisticated 3D design and planning at the dental clinic; and PlanMill, Planmeca's high precision milling unit. You can choose from the comprehensive workflow solution or only those parts that you wish. This is all possible with Planmeca's unique and open interfaces between devices and software.

\section{FLEXIBLE BONDING SOLUTIONS}

VOCO presents several products at BDIA Dental Showcase 2014, such as Futurabond $\mathrm{M}+$, a universal one-component adhesive in bottles.

Futurabond $\mathrm{M}+$ offers the user flexible solutions for every bonding situation. Total-etch, selective-etch or self-etch - the dentist is free to choose and apply the etching technique depending on indication or according to personal preference. Thanks to a new type of monomer technology, overetching of the dentine is impossible. Futurabond $\mathrm{M}+$ is more flexible than conventional bottled bonding agents with regard to its spectrum of application. It offers secure adhesion to various materials such as metals, zirconium or aluminium oxide, as well as silicate ceramics - and requires no additional primer. In combination with Futurabond M+ DCA, the activator available for dual curing purposes, the adhesive is fully compatible with all methacrylate-based self and dual curing composites.

Another highlight is IonoStar Molar, a new glass ionomer restorative material in an application capsule. The material is applied without conditioner or adhesive and scores particularly highly thanks to its non-sticky consistency and perfect marginal adaptation. IonoStar Molar can be modelled immediately after insertion and cures within four minutes. Its lastingly high level of fluoride release counteracts postoperative sensitivity. IonoStar Molar is suitable for restorations of non-occlusion-bearing class I cavities, semi-permanent restorations of class I and II cavities, restorations of cervical lesions, class V cavities, treatment of root caries, restorations of class III cavities, restoration of deciduous teeth, for use as a base or liner and for core build-up as well as temporary restorations.

Visit VOCO on stand F15. www.voco.com

\section{PLACE LOUPES UNDER YOUR MICROSCOPE}

Nuview is the exclusive distributor of Carl Zeiss magnification systems in the UK, and will be on hand at the BDIA Showcase 2014 in London to help delegates explore the extensive range of cuttingedge solutions available.

Visit the team on stand H08 to see demonstrations of the state-of-the-art dental microscopes and loupes they offer.

With a comprehensive range to suit all practices, from the sophisticated OPMI Pico microscope to the convenient Carl Zeis TTL Teleloupe, Nuview is perfectly situated to provide you with the enhanced magnification systems your dental practice needs.

Nuview is proud of its excellent products as well as its reputation for fantastic customer service. With an international network of experienced sales teams and engineers, you can be sure Nuview has the best interests of your practice at heart.

To discover where Nuview's reputation for outstanding service and friendly teams comes from, visit stand H08. www.nuview.co 